Marie-Antoinette et son célèbre dire : deux scénographies et deux siècles de désordres, trois niveaux de communication et trois modes accusatoires

Véronique Campion-Vincent et Christine Shojaei Kawan

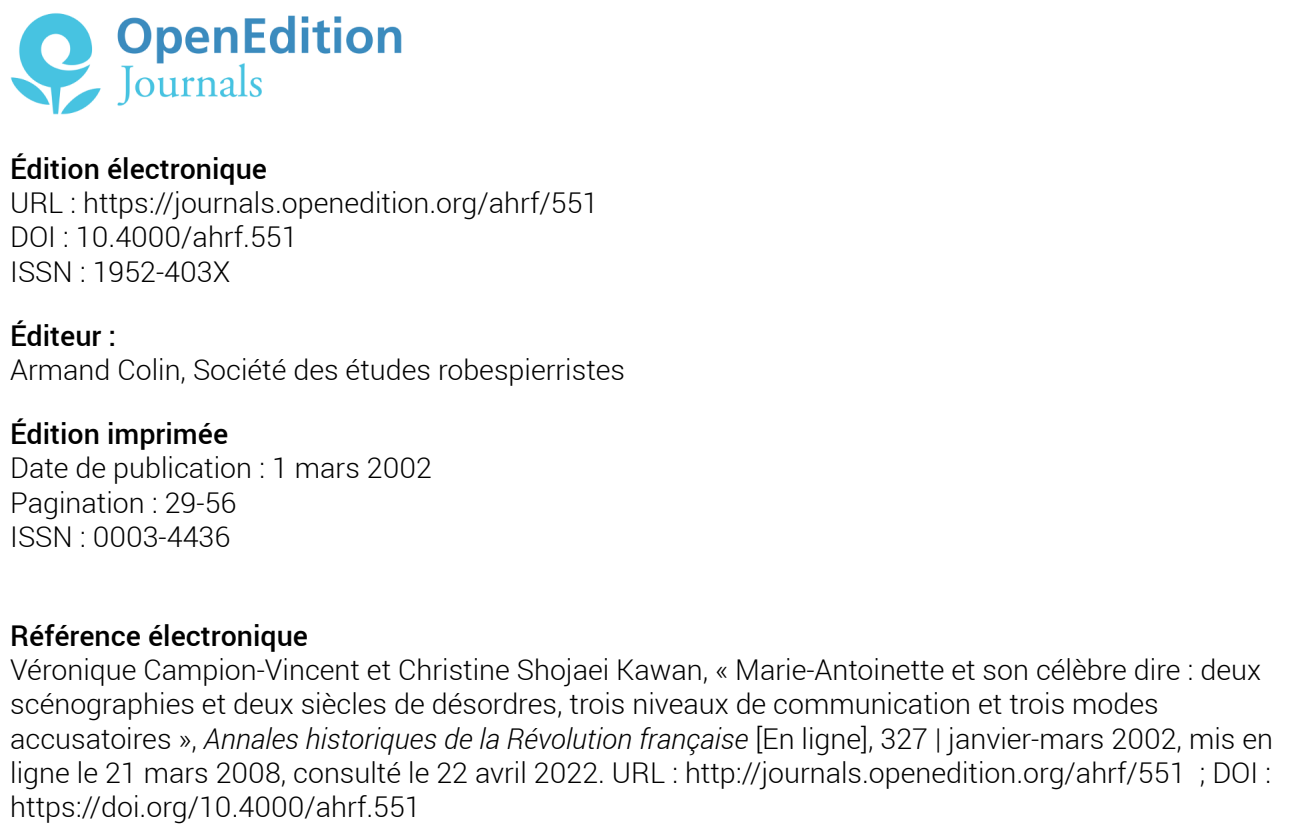




\title{
MARIE-ANTOINETTE ET SON CÉLÈBRE DIRE : DEUX SCÉNOGRAPHIES ET DEUX SIÉCLES DE DÉSORDRES, TROIS NIVEAUX DE COMMUNICATION ET TROIS MODES ACCUSATOIRES (1)
}

\author{
VÉRONIQUE CAMPION-VINCENT ET CHRISTINE SHOJAEI KAWAN
}

\begin{abstract}
Les auteurs étudient le célèbre dire attribué à Marie-Antoinette « lls n'ont pas de pain ? Qu'ils mangent de la brioche ! ", dire également connu sous le titre "Let them eat cake. The queen has been told that peasants have no bread " (conte type 1446 de la classification d'Aarne et Thompson) et proche des proverbes. Elles décrivent les deux scénographies où il apparaît : émeutes de la faim et pique-nique champêtre. Puis elles situent les lieux et périodes de son attestation : I'Allemagne du $\mathrm{XVI}^{\mathrm{e}}$ siècle, la Lettonie de façon récurrente, le $\mathrm{XVIII}{ }^{\mathrm{e}}$ siècle et la Révolution française. L'anecdote est utilisée aux niveaux moyen et folklorique de communication, mais presque totalement absente au niveau savant ; elle décline trois types d'accusations : la bêtise ou l'ignorance mettant en scène des femmes, la cruauté outrageante lorsque les protagonistes sont des hommes. Les auteurs donnent enfin des exemples contemporains de l'utilisation didactique et politique de ce dire, devenu un résumé commode de l'arrogance des puissants et toujours très répandu.
\end{abstract}

Mots clés : famine ; folklore ; Marie-Antoinette ; mots historiques ; stéréotypes.

(1) Des versions antérieures de ce travail ont été (1) présentée au $12^{\mathrm{e}}$ congrès de l'International Society for Folk Narrative Research, Gôttingen, 26-31 juillet 1998, (2) publiée dans Fabula (Berlin) 41, 2000, 13-41.

Nous remercions Christian Amalvi ; François Angelier, Paris ; Karlis Arajs, Riga ; Antoine de Baecque, Paris ; Jôrg Bäcker, Gummersbach ; Robert Benoit, Arras ; la bibliothèque « L'heure Joyeuse », Paris ; John Blahna, Landfall, MN ; Andreas Bode, Munich ; Sabarimuthu Carlos, Bangalore ; Marie-Hélène Degroise, archives municipales de Dijon ; Steven Kaplan, Université de Cornell, NY ; Vivian Labrie, Québec ; JeanPierre Le Goff, Paris; Patricia et Édaïn Lysaght, Dublin; Ulrich Marzolph, Gôttingen; Adrienne Mayor, Princeton, NJ; Wolfgang Mieder, Université de Burlington, VT; Jean-Bruno Renard, Université de Montpellier 3 ; Susanna Sallinen, Turku ; Jurjen van der Kooi, Groningen ; Fionnuala Williams, Belfast.

Annales historiques de la Révolution françaisß2002 -N 1 [29à56] 


\section{Deux scénographies}

Le célèbre dire attribué à Marie-Antoinette apparaît dans deux scénographies : les émeutes de la faim et en particulier les journées d'octobre 1789 ; le pique-nique champêtre :

«Il y a environ cent cinquante ans, les plus pauvres parmi le peuple de Paris marchèrent un jour sur Versailles où habitaient le roi de France et sa femme. C'était une manifestation, vous savez bien ce que c'est. Les pauvres gens se rangèrent devant le château et crièrent: "Nous n'avons pas de pain, nous n'avons pas de pain !" Tant ils étaient miséreux.

La reine Marie-Antoinette regardait par la fenêtre et demanda à un officier :

- Que veulent donc ces gens-là ?

- Majesté, répondit l'officier, ils veulent du pain, ils n'ont pas assez de pain, ils sont trop affamés.

La reine secoua la tête avec étonnement :

- Ils n'ont pas assez de pain ? demanda-t-elle. Mais alors, qu'ils mangent de la brioche!

Vous pensez peut-être qu'elle disait cela pour se moquer des pauvres gens. Non, elle ne savait pas ce qu'est la pauvreté ! Elle pensait que si par hasard on n'a pas assez de pain, on n'a qu'à manger de la brioche. Elle ne connaissait pas le peuple, elle ne connaissait pas la pauvreté, et un an plus tard [2], elle eut la tête tranchée. Tant pis pour elle. » (3)

Cette anecdote pseudo-historique est narrée dans un livre allemand destiné aux enfants, Pünktchen und Anton (1931). Son auteur, Erich Kaestner, était et est toujours très populaire en Allemagne, surtout pour ses romans pour enfants, qui ont été traduits en de nombreux pays. Pûnktchen und Anton, était en 1999 à sa $117^{\mathrm{e}}$ édition, et a été traduit en 25 langues, en France en 1936 (réédition 1952). Sans aucun doute, tout au moins en Allemagne, l'ouvrage a joué un grand rôle dans la diffusion de l'anecdote.

Ce document est la seule version du conte type AaTh 1446 ayant pour protagoniste Marie-Antoinette que l'on puisse véritablement appeler un récit. La reine de France est aujourd'hui tellement associée à ce dire que Stith Thompson décrivit ce conte type : «Qu'ils mangent de la brioche. On a dit à la reine que les paysans manquent de pain». (4) Cependant, parmi les textes qu'il donne comme exemple, on ne trouve ni Marie-Antoinette ni aucune autre reine (5). Il indique dans un ouvrage voisin : «Depuis un siècle

(2) En fait, quatre ans après les événements décrits (5 et 6 octobre 1789, 16 octobre 1793).

(3) Éric KAESTNER, Petit point et ses amis, Paris 1936, 1952, pp. 65-66. Traduction révisée par CSK.

(4) Antti AARNE, Stith THOMPSON, The Types of the Folktale. Second Revision [FF Communications 184]. Helsinki 1961, 1987, type 1446; Thompson ne créa le type 1446 que dans sa deuxième révision, se basant sur le type Estonien 1446* (Antti AARNE : Estnische Märchen - und Sagenvarianten [FF Communications 25]. Hamina 1918) à propos « d'une simple d'esprit », appartenant toutefois à la classe supérieure.

(5) Dans une variante finnoise de 1935 qui n'est pas citée dans le catalogue Aarne/Thompson, une impératrice est la protagoniste, Pirkko-Liisa RAUSMAA Suomalaiset kansansadut. 6: Pilasadut ja kaskut. Helsinki 2000, num. 93. 
et demi, la tradition assure que c'est Marie-Antoinette qui, lorsqu'on lui dit que le peuple manque de pain déclara, "Qu'ils mangent de la brioche" (J2227) [...] Quelles qu'aient été les erreurs de Marie-Antoinette, il est peu probable qu'elle ait proféré cette cruelle remarque ». (6)

Thompson crée donc une abstraction très en harmonie avec le style des contes de fée où figurent surtout des reines anonymes. Il en va de même pour Erich Kaestner qui a simplifié les événements des 5 et 6 octobre 1789, la marche des femmes de Paris à Versailles, pour en faire une scène ressemblant au début d'un conte de fée où rois et reines sont souvent montrés à la fenêtre ou au balcon : on sait que Marie-Antoinette fut appelée au balcon par les manifestants, et qu'elle y fut accompagnée par un "grand officier », le marquis de La Fayette, alors commandant de la nouvelle garde nationale de Paris.

Ce détail indique que, très probablement, Kaestner connaissait les faits historiques et les a changés délibérément pour les adapter à l'anecdote, bien qu'il ne soit pas le seul qui lie le dire attribué à Marie-Antoinette aux journées d'octobre 1789: sous l'intitulé «Sarcasmes », un recueil espagnol de citations datant de 1952 évoque la marche du peuple parisien affamé à Versailles (7); tout en précisant que l'attribution à Marie-Antoinette est probablement fautive.

Enfin, on note en Inde deux variantes curieusement semblables se situant dans le contexte d'émeutes de la faim. La première a été collectée à Bombay au début du $\mathrm{XX}^{\mathrm{e}}$ siècle. Pendant une famine désastreuse dans le Gujarât, le prince héritier voit les foules affamées défiler devant le palais : «Il demande pourquoi ils ne restent pas chez eux en mangeant du khoja et du sucre $\gg .(8)$

Le second récit a été recueilli récemment à Mysore, ville du sud de l'Inde (9) :

«C'est un roi. Il ne s'occupe pas de ses sujets. Il ne comprend pas les difficultés de ses sujets. Il mène une vie de luxe. Il demande à son ministre de s'occuper de l'administration de son royaume. Les ministres sont plus cruels que le roi; ils augmentent les impôts et les gens du pays ont beaucoup de problèmes. Les sujets sont très tristes. Puis une famine survient et le peuple connaît de nouveaux malheurs. D'un côté les ministres posent des problèmes

(6) Stith THOMPSON, The Folktale, Berkeley 1946, 1977, p. 270. Traduction par VCV, comme pour les citations suivantes originellement en anglais.

(7) Vicente VEGA, Diccionario ilustrado de frases célebres y citas literarias, Barcelona 1952, 1973, p. 565 , num. 13 .

(8)Cité d'après Archer TAYLOR «And Marie Antoinette Said...» Revista de etnografla 22 (1968), pp. 1-17, ici 9. Nous n'avons pu consulter ce texte, ni les autres variantes indiennes citées dans Stith THOMPSON, Warren E. ROBERTS : Types of lndic Oral Tales [FF Communications 180]. Helsinki 1960, type 1446.

(9) Recueilli le 5 février 1999. auprès de Mahalakshmi, (femme de 30 ans vivant à Mysore, elle appartient à la caste intermédiaire Kumbara), par Jayalalitha Venkatesh, enseignante de 26 ans, traduit par Sabarimuthu Carlos. 
et de l'autre, les gens n'ont plus rien à manger puisque c'est la famine. Le peuple est forcé de se révolter contre le roi ; le roi a mis de côté beaucoup de grain dans ses greniers. Bien qu'il ait du grain à distribuer au peuple, il n'est pas prêt à le donner au peuple. Incapable d'en supporter davantage, le peuple décide de se soulever contre le roi. Ils crient "À bas le roi ; nous voulons du roti [aliment de base fait à partir de blé]." Le roi connaît-il les difficultés du peuple ? Le roi demande aux ministres "Pourquoi font-ils tant de bruit parce qu'ils n'ont pas de roti? S'il n'y a pas de roti, qu'ils mangent du holige [dessert des jours de fête]." Mais ce qu'il y a de bizarre est que, lorsqu'il n'y a pas de roti, qui donnera du holige ? Mais le roi ne sait rien et s'est oublié dans les plaisirs. Finalement le peuple se soulève contre le roi et le pend à un arbre fait pour ça. Il y a de bons et de mauvais rois en ce monde. Cette histoire montre comment un mauvais roi est puni par le peuple. »

Nous verrons plus tard que les variations de l'anecdote mettant en scène d'autres protagonistes que Marie-Antoinette apparaissent surtout dans le contexte d'émeutes de la faim.

Archer Taylor, dans son article de 1968 sur le dire de Marie-Antoinette et des anecdotes et proverbes proches, suggérait que le récit de Bombay n'était pas influencé par les traditions européennes car il n'associait pas le dire de Marie-Antoinette aux journées d'octobre, mais avait une autre scène en tête :

« Je vois une scène pastorale, où des dames de la cour sont assises sur l'herbe et des nourritures variées abondent. On converse à loisir de la vie, du monde et des plaintes des paysans qui affirment manquer de pain. Une des nobles dames - on suggère que c'est Marie-Antoinette - dit, distraitement ou sans réfléchir ou peut-être sardoniquement, "Pourquoi ne mangent-ils pas de la brioche ?" ou "Qu'ils mangent de la brioche !". » (10)

Malheureusement, Taylor ne peut dire d'où lui vient ce souvenir, mais on peut assumer que cette scène de pique-nique est d'origine américaine ou anglo-américaine.

De toute façon la première scénographie est plus significative puisque les journées d'octobre ont commencé par une émeute de la faim et que le cri de ralliement de la foule ayant ramené la famille royale à Paris était : "Nous ramènerons [...] le boulanger, la boulangère et le petit mitron !. » (11)

Toutefois bien des exemples de l'anecdote attribuée à MarieAntoinette ou à d'autres dames nobles ne sont nullement situées ni narrées ; il s'agit simplement d'une brève réplique.

(10) TAYLOR, op. cit., p. 1.

(11) Jules MICHELET, Histoire de la Révolution française 1, [Paris, 1847] éd. Gérard Walter, Paris, Pléiade, 1952, 1961, p. 256. Autres attestations dans Eugène LABAUME, Histoire monarchique et constitutionnelle de la Révolution française 3, Paris 1835, p. 547 ; Louis B LANC, Histoire de la Révolution française 1-3 Paris, 1847-1852, ici t. 3, p. 254 ; Mémoires sur la vie de Marie-Antoinette, reine de France et de Navarre par Mme CAMPAN, éd. F. Barrière, Paris 1849, p. 254; Michel VOVELLE, La chute de la monarchie, 1787-1792, Paris, 1972, pp. 136-138. 


\section{Deux siècles de désordre}

Le dire est situé principalement dans deux siècles de désordre: La Révolution française; l'Allemagne du $\mathrm{XVI}^{\mathrm{e}}$ siècle; par ailleurs il est récurrent dans la situation de domination des Allemands en Lettonie.

Dans la France pré-révolutionnaire, une période marquée par un appauvrissement général et plusieurs famines entraînant des émeutes, cette réplique était attribuée à plusieurs nobles dames, le plus souvent à la tante par alliance de Marie-Antoinette, Madame Victoire, fille du roi Louis XV (12). Plus tôt, Rousseau avait fait référence à une "grande princesse" anonyme. Quant au dictionnaire Larousse, il attribue la remarque à une "noble hétaïre", probablement une des maîtresses du roi Louis XV, Madame du Barry ou Madame de Pompadour, cette dernière étant aussi considérée comme l'auteur du cynique : «Après nous le déluge ! ». (13)

«Quelquefois, aux justes plaintes du peuple, on répondait par des sarcasmes que les gens bien nés trouvaient fort spirituels. "Il n'a pas de pain, le peuple, disait une noble hétaïre du temps, eh bien qu'il mange de la brioche !" (rubrique Pacte de famine). » (14)

L'attribution du Larousse est située dans un article sur «le pacte de famine », une conspiration supposée de financiers qui auraient organisé, avec la complicité active du pouvoir, une rareté artificielle des céréales, s'enrichissant grâce aux prix élevés tandis que le peuple mourait de faim. Depuis la fin du XIX siècle «le pacte de famine » est considéré comme une rumeur sans fondement, mais il fut longtemps placé dans la catégorie des vérités avérées (15). Dans le contexte des émeutes consécutives aux famines apparaissent des phrases typiques ouvertement cruelles telles que «Qu'ils mangent du foin », «Qu'ils mangent de l'herbe » ou «Qu'ils mangent des trognons de chou ». Alors que la réplique «brioche» ou «croûte de pâté » des nobles dames peut être interprétée comme cynisme, ignorance ou sottise, il ne fait pas de doute que les dires prêtés aux puissants expriment un mépris total.

(12) Giuseppe FUMAGALLI, Chi l'ha detto ? Tesoro di cilazioni italiane e straniere di origine letteraria e storica, Milano, 1915, num. 1333; Pelle HOLM, Bevingade ord och andra talesatt Stockholm, 1961, 40. TAYLOR (op. cit.), p. 5 cite Holm.

(13) Le nouveau Petit Robert, Paris 1993, p. 263 (également attribué à Mme du Barry); Vega (op. cit.) p. 118, num. 6; Franz Freiherr VON LIPPERHEIDE, Spruchwônerbuch Berlin, 1934, p. 801; Jochen KLAUSS, Charlotte von Stein. Die Frau in Goethes Nähe Zurich, 1995, p. 11. En France, la réplique est plus fréquemment attribuée à Louis XV, Dictionnaire de l'Académie française 1, Paris, 1992, p. 616; Paul-Emile LITTRÉ, Dictionnaire de la langue française 2, Chicago, 1982, p. 1546; François BUCHE Dictionnaire des mots historiques, Paris, 1992, pp. 33 sq. (Littré et Bluche indiquent que l'auteur véritable de la réplique est Mme de Pompadour). Dans le film « Marie-Antoinette» (U.S.A., 1938), Louis XV prononce cette réplique.

(14) Pierre LAROUSSE, Grand dictionnaire universel du XX' siècle 8, Paris 1872, pp. 80-82, citation p. 80

(15) Steven L. KAPLAN Le complot de famine : histoire d'une rumeur au XVIII siècle, Paris, 1982. 
Une autre période de bouleversements de l'histoire européenne, très comparable à la Révolution française, a été le $\mathrm{XVI}^{\mathrm{e}}$ siècle, l'âge de la Réforme. Cette révolution religieuse, basée sur le choix personnel et la liberté de conscience de l'individu a également eu d'importantes conséquences politiques, économiques et sociales. De plus, la guerre des paysans (1524-1525) qui se déroula lors de son premier âge ainsi que les tendances révolutionnaires du mouvement anabaptiste ont été considérées comme annonçant les révolutions française et communiste.

On ne s'étonnera donc pas de trouver dans des textes allemands de cette époque des attestations anciennes de la réplique attribuée ensuite à MarieAntoinette : dans le recueil de plaisanteries de Martin Montanus Das Ander theyl der Garten gesellschafft (16), et dans Epitome Historiarum du prédicateur protestant Wolfgang Bûtner, parmi les exempta concernant l'usure et l'avarice (17). La scénographie est analogue aux journées d'octobre : des gens du peuple affamés implorent une princesse ou une noble dame tandis qu'une autre se demande pourquoi ils ne mangent pas du pain et du fromage. Tout comme dans la France pré-révolutionnaire, nous trouvons dans plusieurs compilations de prédicateurs luthériens des $\mathrm{XVI}^{\mathrm{e}}$ et $\mathrm{XVII}{ }^{\mathrm{e}}$ siècles des phrases ouvertement cruelles adressées au peuple frappé par la famine, phrases encore plus méprisantes que les phrases françaises du XVIII ${ }^{\mathrm{e}}$ siècle puisque la forme allemande ancienne est «Qu'ils mangent de la merde » (18).

Un troisième pays où les présentations narratives du conte type sont attestées est la Lettonie. Ici, la classe dominante était les Allemands qui monopolisaient les privilèges et le pouvoir. Jusqu'au milieu du XIX ${ }^{e}$ siècle les paysans lettons n'avaient pas le droit d'acquérir ou de posséder de la terre, et lorsque finalement ils y furent autorisés, la plupart d'entre eux ne purent en acheter assez pour devenir autonomes. En Lettonie, nous trouvons des anecdotes orales critiquant la classe supérieure allemande où un membre de l'aristocratie terrienne allemande, en général la dame du manoir ou une baronne (parfois aussi le propriétaire ou sa fille, dans un cas un pasteur), déclare aux paysans affamés qu'ils devraient manger du pain blanc nappé de beurre, ou réchauffer les restes de viande de la veille. Ces récits se réfèrent à une situation sociale ayant perduré plusieurs siècles et ne sont pas situés dans le temps, sauf un récit où une dame, lorsque le pain est rationné durant la Première Guerre mondiale, affirme que les gens devraient cuire des gâteaux ; une seule variante renvoie à une personne précise (la maîtresse du domaine d'Aumeistari) (19).

(16) Martin MONTANUS, Schwankbücher, éd. Johannes Boite, Tubingen, 1899, [1559, 1566], p. 299, $\mathrm{n}^{\circ} 48(50)$

(17) Wolffgangum BUETNERN, Epitome Historiarum, Das ist: Christliche und kurtze beschreibung vieler denchvirdiger Historien vnd Exempel, Leipzig, 1596 [1576], p. 301, n 68.

(18) « Sie solten kott essen » ou « Sie solten Koth essen ».

(19) K. ARAJS, A. MEDNE, Latvie upasaku tipu raditajs [Types des contes lettons], Riga, 1977, type 1446. En 1994, Guntis Pakalns, Riga, narra à CSK une autre version que son père lui avait racontée. Le protagoniste est ici le fils du propriétaire allemand. Voir aussi la variante estonienne citée par Aarne (op. cit. ). 
Suivant Alphonse Karr, le dire de Marie-Antoinette est attesté dans «un livre daté de 1760 où on raconte le même mot d'une duchesse de Toscane (20)»; transformée en «princesse piémontaise», cette noble italienne se retrouve en Irlande (21). En Italie, le poète patriotique Francesco DalPOngaro (1808-1873) a utilisé l'anecdote pour un de ses «couplets » politiques; elle est narrée, dans le style abstrait d'un conte de fée, à propos d'un couple royal anonyme, et introduite par la formule courante : «Il était une fois un roi et une reine (22)». Nous avons vu que les variantes indiennes attribuent également des remarques du type «brioche » à des rois et princes héritiers anonymes. En Inde, une influence coloniale est possible, voire probable, mais des versions de AaTh 1446 sont également attestées aussi loin qu'en Chine, où l'on ne saurait parler d'influence du colonisateur. L'une met en scène un jeune homme riche anonyme, une autre l'empereur Hui de la dynastie occidentale Jin (291-306 A.D.), et évoque les plaisanteries stigmatisant le caractère trop éthéré des érudits confucéens :

«Une fois, alors que l'empereur Hui de la dynastie Jin mangeait de la viande pendant un banquet impérial, le gouverneur général de l'Est lui annonça qu'il y avait une grande famine à l'Est, et que beaucoup d'affamés avaient péri. L'empereur dit: "Si ces affamés n'ont pas de céréales, ils devraient manger de cette viande. Cela remplit également l'estomac - pourquoi mourir de faim ? " » (23)

L'anecdote, expurgée de tout contexte social, persiste en Chine. Ainsi dans ce recueil contemporain de plaisanteries :

«La décoction de Ginseng. Un jeune homme de riche famille vit un jour un pauvre porteur couché sur le sol tandis qu'il marchait le long d'une route un matin. Il demanda aux passants, "Pourquoi cet homme est-il couché là ?"

On lui répondit: "Il n'a aucune nourriture pour se remplir l'estomac et a une telle faim qu'il se couche sur le sol pour retrouver son souffle."

Le jeune homme riche dit : "Puisqu'il n'a rien à manger, pourquoi n'a-t-il pas pris une tasse de décoction de ginseng avant de partir de chez lui le matin ? Cela l'aurait nourri pour une demi-journée". » (24)

(20) Alphonse KARR, Les guêpes, Paris (mars 1843), pp. 85-86.

(21) S. O SUILLEABHAIN, R.-T. CHRISTIANSEN, The Types of the Irish Folktale [FF Communications 188], Helsinki 1967, num. 1446 (1 référence).

(22) Francesco DALL'ONGARO, Stomelli, poemetti e poésie, ed. Nico Schileo, Treviso 1912, p. 7. Fumagalli (op. cit.) cite, comme source possible ou analogue du stomello de Dall'Ongaro, MarieAntoinette, Madame Victoire, Foulon et la grande princesse de Rousseau, mais ne mentionne nulle princesse italienne. Taylor (op. cit.), discute en détail le poème de Dall'Ongaro et Fumagalli.

(23) Nai-Tung TTNG, A Type Index of Chinese Folktales in the Oral Tradition and Major Works of NonReligious Classical Literature [FF Communications 223], Helsinki, 1978, type 1446, cite deux vanantes chinoises.

(24) Selected Jokes from Past Chinese Dynasties, vol. 2, Beijin, 1992, pp. 92-93. La décoction de ginseng est très onéreuse. 


\section{Trois niveaux de communication}

Trois niveaux, savant, moyen et folklorique utilisent l'anecdote. On notera une absence presque totale au niveau savant.

Il semble logique de supposer qu'un dire célèbre, utilisé comme moyen de propagande contre Marie-Antoinette, ait été mentionné par ses nombreux biographes. Par ailleurs, Archer Taylor a écrit que l'attribution du dire à Marie-Antoinette avait été rejetée par les historiens (25). Toutefois Christine Shojaei Kawan, lorsqu'elle préparait un article sur le conte type AaTh 1446 (26), découvrit avec étonnement qu'aucune allusion n'y était faite dans les biographies de Marie-Antoinette (27) ni dans les nombreux ouvrages historiques sur la Révolution française qu'elle consulta, sauf dans l'ouvrage de Georges Lefebvre où le dire est rejeté comme erroné (28), même lorsque sont mentionnés ou étudiés les nombreux pamphlets, satires, chansons et épigrammes mis en circulation contre la reine (29).

Elle demanda donc de l'aide à Véronique Campion-Vincent, qui mena une recherche systématique parmi les sources possibles, dont deux étaient contemporaines de la Révolution: les caricatures, libelles et pamphlets contre Marie-Antoinette d'une part, la presse révolutionnaire de l'autre. Une troisième source, les manuels scolaires, date du XIX ${ }^{\mathrm{e}}$ siècle.

Même avant que n'éclate la Révolution, Marie-Antoinette, très impopulaire auprès du peuple mais aussi auprès de nombreux aristocrates, était accusée de toutes sortes de désordres : ivrognerie, indécence, participation à des orgies, inceste. Décriée par les libelles et pamphlets, la reine partage son temps entre le gaspillage d'immenses sommes et la participation à de scandaleuses orgies; elle est donc présentée comme une participante active à l'appauvrissement du peuple. Toutefois, pas une ligne dans ces documents ne lui attribue le célèbre dire (30). De même, les historiens travaillant sur la presse révolutionnaire contactés par VCV n'indiquèrent aucune référence à l'histoire de la brioche dans leurs sources (31). De plus, la consultation de

(25) TAYLOR, op. cit., p. 4.

(26) Christine SHOJAEI KAWAN, «Kuchen : LaBt sie K. essen! (AaTh 1446)», dans Rolf Wilhelm Brednich et al. [eds], Enzyklopädie des Märchens 8, Berlin/New York, 1996, pp. 536-541.

(27) Stefan ZWEIG, Marie Antoinette. Bildnis eines mittleren Charakters, (Leipzig 1932) Frankfurt am Main, 1990, pp. 186-188 ; Edmond et Jules DE GONCOURT, Histoire de Marie-Antoinette, Paris, 1859. La remarque est citée comme erronée dans : Joan HASUP, Marie Antoinette, New York, 1987, p. 75 ; Vincent CRONIN, Louis and Antoinette, London, 1989, p. 13.

(28) Georges LEFEBVRE, La Grande Peur de 1789, Paris, 1932, p. 34.

(29) BLANC, op. cit., t. 2, pp. 22, 23, 35., t. 3, pp. 158-169; GONCOURT, op. cit., pp. 178-182; ZWEIG, op. cit., pp. 181, 188-193, 244-246.

(30) Chantai THOMAS, La reine scélérate. Marie-Antoinette dans les pamphlets, Paris, 1989 ; Henri DALMERAS, Marie-Antoinette et les pamphlets royalistes et révolutionnaires, Paris, 1907, pp. 45, 236, 238-240.

(31) VCV consulta Antoine de Baecque, qui a beaucoup écrit sur la période et est notamment l'auteur de La caricature révolutionnaire, Paris, 1988, et contacta par téléphone (7 novembre 1994) Marianne Giudicelli-Darras qui menait une étude sur la presse révolutionnaire pendant les journées d'octobre 1789. 
l'étude de Christian Amalvi sur la présentation des personnages historiques dans les manuels français scolaires du primaire de 1833 à 1919 montra que rien n'est trouvé parmi ces matériaux (32). L'étude contient toutefois une intéressante analyse de l'image négative de Marie-Antoinette :

«Les hommes disposent dans la galerie des hommes illustres de l'histoire de France d'une confortable majorité de places. De surcroît ces places sont le plus souvent les plus importantes tandis qu'aux femmes sont réservés des rôles peu flatteurs: [...] la frivole et coquette Marie-Antoinette est souvent présentée comme la responsable des malheurs de la France à la fin du xvme siècle [...]. Bref, en apparence les femmes de l'histoire de France dans les manuels primaires, qu'ils soient catholiques ou laïques, ne semblent pas jouer un rôle de premier plan, excepté celui de bouc émissaire, en particulier réservé aux femmes étrangères qui cumulent en quelque sorte tous les caractères négatifs attribués d'ordinaire aux "héros" malfaisants ; ainsi la trahison de "linfâme Isabeau de Bavière" s'explique à la fois par sa nationalité et son sexe; il en va de même pour Catherine et Marie de Médicis et surtout pour Marie-Antoinette. Et le double caractère national et sexuel qu'elles incarnent se résume en un adjectif féminin qui doit les qualifier une fois pour toutes : en effet la plupart des manuels n'omettent pas de faire précéder les noms d'Isabeau de Bavière, de Catherine de Médicis et de Marie-Antoinette des épithètes peu élogieuses d'allemande, d'italienne et d'autrichienne. » (33)

Il est évident que bien que le dire en tant que tel ne soit pas présent dans les manuels scolaires, le portrait qu'ils tracent de Marie-Antoinette est celui d'une femme qui aurait bien pu faire une telle remarque frivole, vaine et stupide.

À l'opposé, dans un ouvrage très hagiographique sur Marie-Antoinette, Otto Friedrichs énumère de nombreux actes de générosité de la reine et lui prête une démarche auprès de Louis XVI, en août 1774, afin que le monopole des grains ne soit pas renouvelé (34).

Alors que le dire célèbre de Marie-Antoinette est absent des ouvrages d'historiens et des sources contemporaines, et en général aussi des ouvrages de référence (35), il est aisément trouvé dans les dictionnaires de cita-

(32) Christian AMALVI, La galerie des hommes illustres de l'histoire de France dans l'enseignement primaire 1-7, Thèse, Paris, 1977. Contacté par téléphone, l'auteur indiqua à VCV que ses sources étaient muettes sur ce dire. paginé).

(33) AMALVI, op. cit., tome 7, appendice 2 « La vision de la femme dans les manuels d'histoire » (non

(34) Otto FRIEDRICHS, Marie-Antoinette calomniée. La "grande calomnie du XVII siècle» défendue contre les libelles immondes qui salirent en elle la femme, l'épouse, la mère, Paris, Maurice d'Hartoy, 1948, p. 323. L'auteur appuie son affirmation sur une lettre de Mercy-Argenteau, ambassadeur d'Autriche, à l'impératrice Marie-Thérèse.

(35) Deux exceptions sont The New Enyclopaedia Britannica, Micropaedia 6,1983, pp. 620 et suiv. (décrit le dire comme attribué à la reine par les agitateurs révolutionnaires alors que selon nos recherches l'attribution doit être plus tardive) ; The Macmillan Dictionary of Women's Biography. London/Basingstoke, 1989 [1982], p. 357 (note que «la fameuse remarque "qu'ils mangent de la brioche" est probablement apocryphe »). 
tions (36). Ceux-ci affirment souvent que le dire est attribué à tort à la reine par ses ennemis. Les dictionnaires français citent le dire, mais ne l'attribuent pas (37).

La première attestation écrite que nous ayons trouvée liant MarieAntoinette à ce dire célèbre apparaît cinquante ans après sa mort, en 1843, dans les écrits d'Alphonse Karr qui le présente comme un bruit lancé contre la reine par ses ennemis :

«On se rappelle quelle indignation on excita, dans le temps, contre la malheureuse reine Marie-Antoinette, - en faisant courir le bruit - que, entendant dire que le peuple était malheureux et qu'il n'avait pas de pain, - elle avait répondu : "eh bien ! qu'il mange de la brioche". » (38)

Les attestations écrites semblent rares jusqu'au début du $\mathrm{XX}^{\mathrm{e}}$ siècle (39). Il semble donc qu'au XIX ${ }^{\mathrm{e}}$ siècle l'anecdote attribuée à MarieAntoinette se soit transmise oralement alors qu'au $\mathrm{XX}^{\mathrm{e}}$ siècle, et surtout depuis les années trente, elle ait été répandue par des ouvrages littéraires tels que le livre pour enfants de Kaestner Pünktchen und Anton.

Les récits et anecdotes de brioche ou de cake attribués à MarieAntoinette et à d'autres personnalités, anonymes ou non, sont des perceptions populaires de l'histoire, exprimant une critique sociale et/ou un procédé de caractérisation de figures historiques. Cette interprétation populaire de l'histoire forme le niveau moyen.

À un troisième niveau, le niveau folklorique, on trouve les proverbes ainsi qu'une veine plaisante, drôle, sans conséquence et d'une joyeuse légèreté.

Archer Taylor a discuté en détail des proverbes proches du dire de Marie-Antoinette (40). On les trouve en de nombreuses langues et ils sont attestés depuis le Moyen Âge. Toutefois les anthologies de proverbes ne renseignent guère quant à leur usage, ainsi il est difficile de savoir si la pâtis-

(36) Edward LATHAM, Famous Sayings and Their Authors. A Collection of Historical Sayings in English, French, German, Greek, Italian, and Latin, London, 1904, p. 175 ; Renie GEE, Who Said That ?, ed, Graham Donaldson, Maris Ross, London, 1989, p. 99; Bloomsbury Dictionary of Quotations ed. J. Daintith, A. Stibbs, E. Wright, D. Pickering, London, 1987, 1991, p. 250; The Oxford Dictionary of Quotations, ed. A. Partington, Oxford/New York, 1941, 1992, p. 446; FUMAGALLI, op. cit.; VEGA, op. cit.; voir aussi Notes and Queries, 12,5 (1919), pp. 53, 162 et suiv. ; Lutz RÖHRICH, Das groBe Lexikon der spichwörtlichen Redensarten 2, Freiburg im Breisgau/Basel/Wien 1992, p. 898. La référence suivante nous a été signalée par Wolfgang Mieder : Paul F. Jr BOLER, John GEORGE, They Never Said It. A Book of Fake Quotes, Misquotes, and Misleading Attributions, New York/Oxford, 1989, pp. 97 et suiv.

(37) Dictionnaire de l'Académie française 1, Paris, 1932, p. 173 ; Paul ROBERT, Dictionnaire alphabétique et analogique de la langue française 1, Paris 1951, p. 561 ; Jean GIRODET, Dictionnaire de la langue française, Paris 1976, p. 294.

(38) KARR, op. cit., p. 85. op. cit.

(39) Les attestations suivant Karr que nous avons trouvées sont pour la plupart citées par Taylor,

(40) TAYLOR, op. cit., pp. 10-7; Walter GOTTSCHALK, Die bildhaften Sprichwörter der Romanen Heidelberg, 1936, p. 86. 
serie opposée au pain, torta, biscuits ou croûte de pâté, est perçue comme un substitut inférieur ou supérieur. Prenons, par exemple, les exemples cités dans l'anthologie de proverbes allemands de Wander (41): on y trouve «Certains mangent des gâteaux parce que le pain est trop cher » (42) et «Mangez des gâteaux tant qu'il n'y a pas de pain» (43), mais aussi « Des gâteaux rassis valent mieux que pas de pain du tout»(44), ou «Quand les gâteaux font défaut, le pain a bon goût »(45). L'illustration d'un proverbe symétriquement opposé «À défaut de pain, les gâteaux sont bons », se trouve dans une lithographie française, datant probablement du XIX ${ }^{\mathrm{e}}$ siècle, un cas rare de «gâteaux» au lieu de «brioche » ou «croûte de pâté » qui montre un intellectuel en guenilles volant un gâteau tandis que le boulanger lui ôte sa perruque (46).

La célèbre citation de la réplique de la brioche par Rousseau peut être évoquée comme exemple d'utilisation proverbiale dans un mode sans conséquence et drôle. Rousseau raconte qu'il a «emprunté » (c'est-à-dire volé) quelques bouteilles de vin à la maison où il est employé comme précepteur et aimerait ajouter du pain à sa consommation de vin. Mais il est trop élégamment vêtu pour entrer dans une boulangerie. «Enfin je me rappelai le pis-aller d'une grande princesse à qui l'on disoit que les paysans n'avoient pas de pain, et qui répondit "Qu'ils mangent de la brioche !" ». Et il achète de la brioche chez un pâtissier (47). La réplique est citée sans indignation ni condamnation, et il n'y a pas d'intention didactique. Les dictionnaires de citations mentionnant le dire se réfèrent souvent à la «grande princesse » de Rousseau, dont le «pis-aller» est fréquemment déclaré thoughtless [étourdi], ou encore affirment que Rousseau prêtait le dire à MarieAntoinette (48). On sait que Rousseau écrivit la première partie des Confessions (Livres 1-6) en 1765, et ne peut donc avoir pensé à MarieAntoinette qui n'épousa le futur Louis XVI qu'en 1770. Le dictionnaire de

(41) Karl Friedrich Wilhelm WANDER, Deutsches Sprichwörter-Lexikon 2, Leipzig, 1867, Augsburg, 1987, pp. 1657 sq.

(42) WANDER, op. cit., num. 26, avec une variante russe ; num. 28, de Lucerne, Suisse.

(43)Ibid., num. 23, avec une variante hollandaise; num. 24, avec une variante espagnole; GOTTSCHALK^ip. cit.

(44) Ibid., num. 21, avec une variante tchèque.

(45) Ibid., num. 48, avec une variante polonaise ; num. 40, avec une variante anglaise. Au sujet des préférences fluctuantes, voir aussi $n^{\circ} 34$, avec variantes russe et tchèque; $n^{\circ} 35 ; n^{\circ} 50$ avec variante italienne.

(46) Lithographie de Longlumé, Europâisches Brotmuseum, Ebergoetzem.

(47) Jean-Jacques ROUSSEAU, Les Confessions, livre sixième, Paris, Pléiade, 1959 [Genève 1782], p. 269

(48) Wolfgang MIEDER, A Dictionary of American Proverbs, New York/Oxford, 1992, p. 68, n' 14 cite à tort Rousseau comme ayant attribué le dire à Marie-Antoinette ; Burton STEVENSON, The Macmillan Book of Proverbs, Maxims, and Famous Phrases, New York, 1948, pp. 274 et suiv. ; Nigel REES, Cassell Companion to Quotations, London, 1997, p. 380 [suggère que Marie-Antoinette « citait » peut-être une remarque antérieure] ; Gregory TTTELMAN, Random House Dictionary of Popular Proverbs \& Sayings, New York, 1996, p. 211, pour lequel Rousseau se rappelle le «dire étourdi d'un grand prince». Sans donner de références, Titelman mentionne une anecdote sur un empereur chinois [L'empereur Hui de la dynatie Jin, anecdote citée page 3]. 
Bluche affirme que le dire est attribué à tort à Marie-Antoinette et cite longuement Rousseau (49).

Le contexte dans lequel le roi Louis XVIII cite son ancêtre MarieThérèse d'Autriche nous rappelle le dicton allemand «Lorsque la misère est grande, la saucisse a encore bon goût même s'il n'y a pas de pain » (50) : pendant leur fuite de France, Louis et son compagnon s'arrêtèrent à Vaurains, entre Soissons et Laon.

«Alors je proposai de déjeuner; nous avions un pâté et du vin de Bordeaux; mais nous avions oublié d'avoir du pain. Aussi, en mangeant la croûte avec le pâté, nous songeâmes à la reine Marie-Thérèse, qui répondit un jour que l'on plaignait devant elle les pauvres gens qui n'ont pas de pain : "Mais, mon Dieu, que ne mangent-ils de la croûte de pâté ?" » (51)

L'anecdote, ici, ressemble à une plaisanterie de famille. L'attribution par Louis XVIII à son aïeule, Marie-Thérèse d'Autriche, l'épouse de Louis XIV, est peut-être due à une analogie entre les deux reines «autrichiennes »; également au fait qu'il ne pouvait attribuer une remarque désobligeante à Marie-Antoinette devenue une martyre, bien que, dans sa jeunesse, il l'eût cordialement détestée (52).

Latham affirme à tort que Louis XVIII attribue l'anecdote à MarieAntoinette (53). La croûte de pâté apparaît comme «caisse [sic] de pâté » dans Notes and Queries où ce dire est lié à Marie-Antoinette (54). Un proverbe hollandais du XVI siècle cité par Taylor suggère que l'anecdote de la croûte de pâté peut remonter à une période plus ancienne que celles de Marie-Thérèse d'Autriche ou de Madame Victoire.

Les modifications de répliques célèbres sont courantes et peuvent en changer complètement le sens, comme illustré par une plaisanterie française où le sens de la réplique est plaisamment inverse - les pauvres réclament du gâteau, mais les riches ne sont prêts à donner que du pain - et qui jouent sur les conceptions bourgeoises de conduite appropriée, sans toutefois la moindre critique sociale :

« - Madame, dit le clochard à la porte de l'église, donnez-moi un peu d'argent que je puisse m'acheter un gâteau. - Un gâteau ? Mais enfin, quelqu'un comme vous ferait mieux de manger du pain... - Peut-être, madame, mais aujourd'hui, c'est mon anniversaire. » (55)

(49) BLUCHE, op. cit., 129

(50) Ce proverbe bien connu (CSK le connaissait depuis son enfance et plusieurs de ses collègues le lui ont cité) n'a pas été retrouvé dans les recueils de proverbes allemands consultés.

(51) [Louis-Stanislas-Xavier de France] Relation d'un voyage à Bruxelles et à Coblentz (1791), Paris, 1823 , p. 59

(52) Lorsqu'il était encore Comte de Provence, Louis XVIII avait fortement intrigué contre la reine Marie-Antoinette et faisait partie de ceux qui avaient fait circuler sur elle des chansons, pamphlets, et épigrammes scandaleux.

(53) LATHAM, op. cit.

(54) Notes and Queries 12, 5, London, juin 1919, p. 163.

(55) Hervé NEGRE, Dictionnaire des histoires drôles, Paris, 1967, n 547 
Dans les pays anglophones, et particulièrement aux États-Unis, la réplique est extrêmement répandue (56). Certaines de ses variantes se réfèrent à Marie-Antoinette ou à un arrière-plan révolutionnaire : telle cette publicité Hennessy vaguement évocatrice de la scène du pique-nique (fig. 1) ; deux caricatures de Marie-Antoinette conduite à l'échafaud, l'une où elle tente de se justifier, l'autre où le bourreau la félicite pour son excellente réplique (fig. 2 et 3 ); ou encore un dessin montrant un roi s'envolant vers l'exil (fig. 4) - très dans le style de Friedrich August III de Saxe dont on dit qu'il abdiqua en 1918, en déclarant « Démerdez-vous vous-même (57) »; ou une publicité pour un service de messagerie montrant un message à la reine de France «Marie-Antoinette - les paysans s'agitent. Ne parlez pas de "brioche". Faites-moi confiance » (58). Toutefois les allusions historiques sont en minorité. Les tendances dominantes sont, d'une part une interprétation littérale de la réplique comme le montrent les multiples publicités pour des produits de pâtisserie, des services traiteur ou des aliments allégés (59), la discussion de problèmes nutritionnels (60) ou des titres de manuels de cuisine (61); d'autre part, la réduction à la formule ouverte «Qu'ils mangent de ...» rend la réplique applicable non seulement à tout type d'aliment (62), mais encore à virtuellement tout sujet (63).

(56) Les conclusions que nous avons tirées des nombreux matériaux concernant le dire de MarieAntoinette qui nous ont été transmis par Wolfgang Mieder à partir de sa collection ont pu être corroborées par une recherche sur Internet qui a donné des résultats très abondants.

(57) « Macht euern Dreck alleene. » Au sujet de cette anecdote bien connue, voir les sites www.mdr.de/geschichte/inhalt.html (film de Christian Frey «Fûrstenzug und Montagsdemo », diffusé le 14 novembre 1999); www.tagesspiegel.de/archiv/1999/04/05/ku-hi-13648.html; www.bistum-wuerzburg.de/ medien/Sobla 13/Jesustitell3.html.

(58) Publicité parue dans US Airways Attaché, octobre 1998, p. 9.

(59) Par exemple un sosie d'Élisabeth d'Angleterre se régalant de biscuits allégés (Modem Maturity, June-July 1992), à Chicago une chaîne de boulangeries «inspirées de Marie-Antoinette» intitulée «Chicagoland Let Them Eat Cake» (centerstage.net/chicago/restaurants/cake/66washington.html); «la recette originale du célèbre savarin au rhum de Marie-Antoinette » vendue par Lisé Saint-Amour, Severna Park, MD (www.freeway.fr/MARIE-ANTOINETTE/cake.html) ; les gâteaux d'anniversaire du traiteur de Stanford University Dining Services (www.stanford.edu/dept/hds/dining/special/cake.html).

(60) Ronald E. KLENMAN, Let Them Eat Cake! The Case against Controlling what Your Children Eat. The Pediatrician's Guide to Safe and Healthy Food and Growth, New York, 1994; « Let Them Eat Cake. A comparison of desirable weight tables » Time, 19 août 1985, 57 ; « Anorexia expert Dr Dee Dawson told Jennie Bristow why we should not deprive children of fatty foods : Let them eat cake - and crisps, chips and chocolate » (www.informinc.co.uk/LM/LM107/LM107-Cake.html).

(61) Susan Gold PURDY, Let Them Eat Cake. 140 Sinfully Rich Desserts - with a Fraction of the Fat, New York, 1997 ; Virginia N. WHITE, Let Them Eat Cake and Other Low-Fat and Low-Cholesterol Desserts, Minneapolis, 1992.

(62) «Bread for China: Let them eat shortcake» Time, $1^{\text {er }}$ septembre 1980, 49 (ouverture d'une boulangerie à l'américaine de démonstration à Beijing) ; « Let them eat fromage ! » Smithsonian Magazine, novembre 1996 [www.smithsonianmag.si.edu/smithsonian/issue.../fromage.htm] ; « Let them eat. crustless sandwiches : Public can join corporate shareholders for free lunch » (www.straightgoods.com/item51.asp ; april 10, 2000).

(63) Joseph HOFFMAN, Let Them Eat Cake, Holt, Minn., 1938 [poems] ; Colin David (ed.) WEBB Let Them Eat Cake. Writers against Victorian Values Millbrook et al., 1986 [political poetry]; Council of Social Service of the A.C.T. : Let Them Eat Cake... A Profile of Poverty in Canberra, Canberra, 1986; Peter TOWNSEND, Let Them Eat Cake. The Full Text of Peter Townsend and David Gordon from the New Review no. 4, London, 1991 (au sujet de la Sécurité sociale et des aides publiques); Frederick LONSDALE, Let Them 


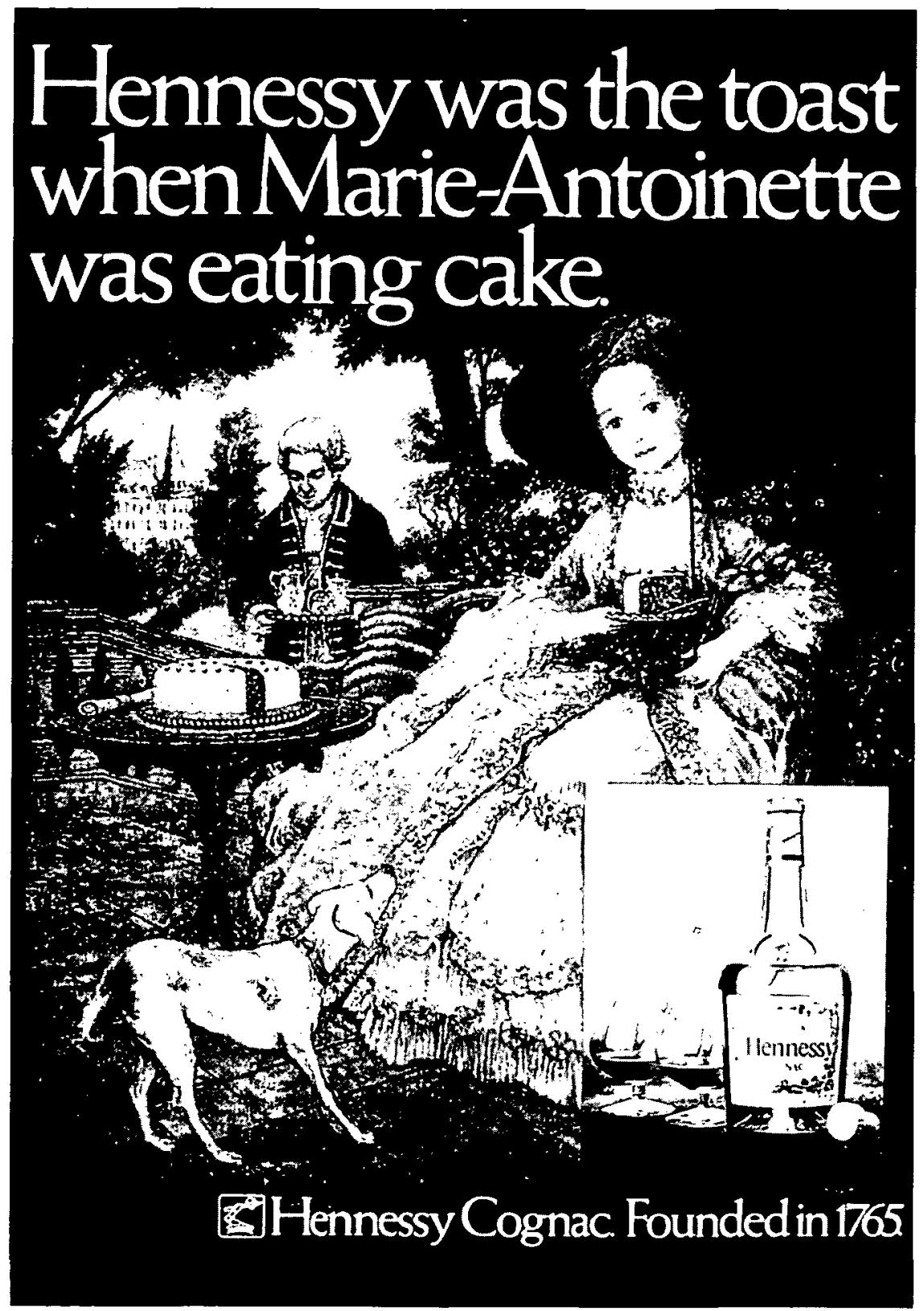

Fig. $1:$ «Hennessy was the toast when Marie-Antoinette was eating cake », Punch (17 octobre 1979) $656 \mathrm{~d}$; ibid. (9 juillet 1980) IV ${ }^{\mathrm{e}}$ de couverture (collection Wolfgang Mieder) 


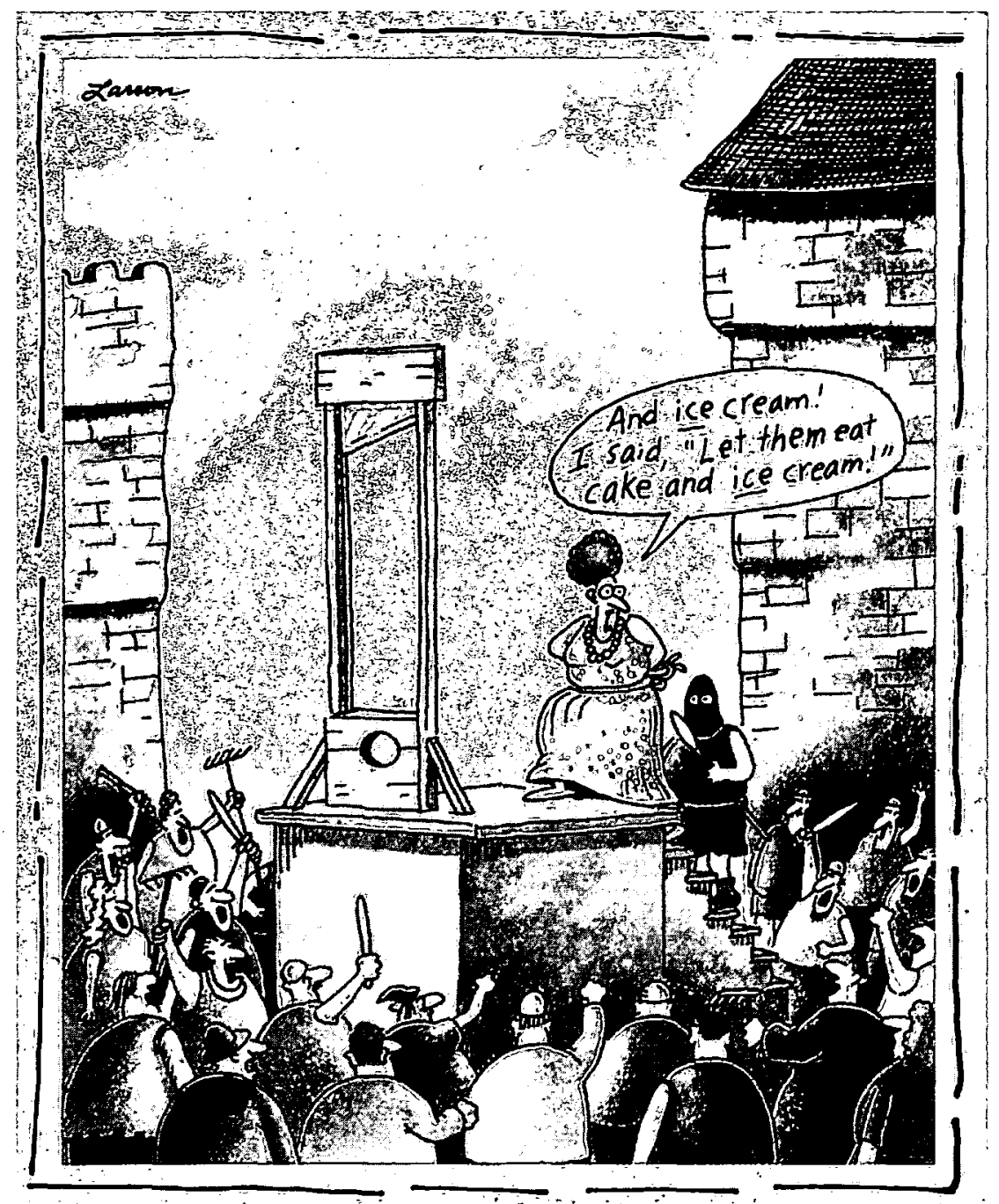

Marie Antoinette's last-ditch effort to save her head.

Fig. 2 : «Marie Antoinette's last-ditch effort to save her head», Gary Larson, carte postale (Paris, octobre 2001). 


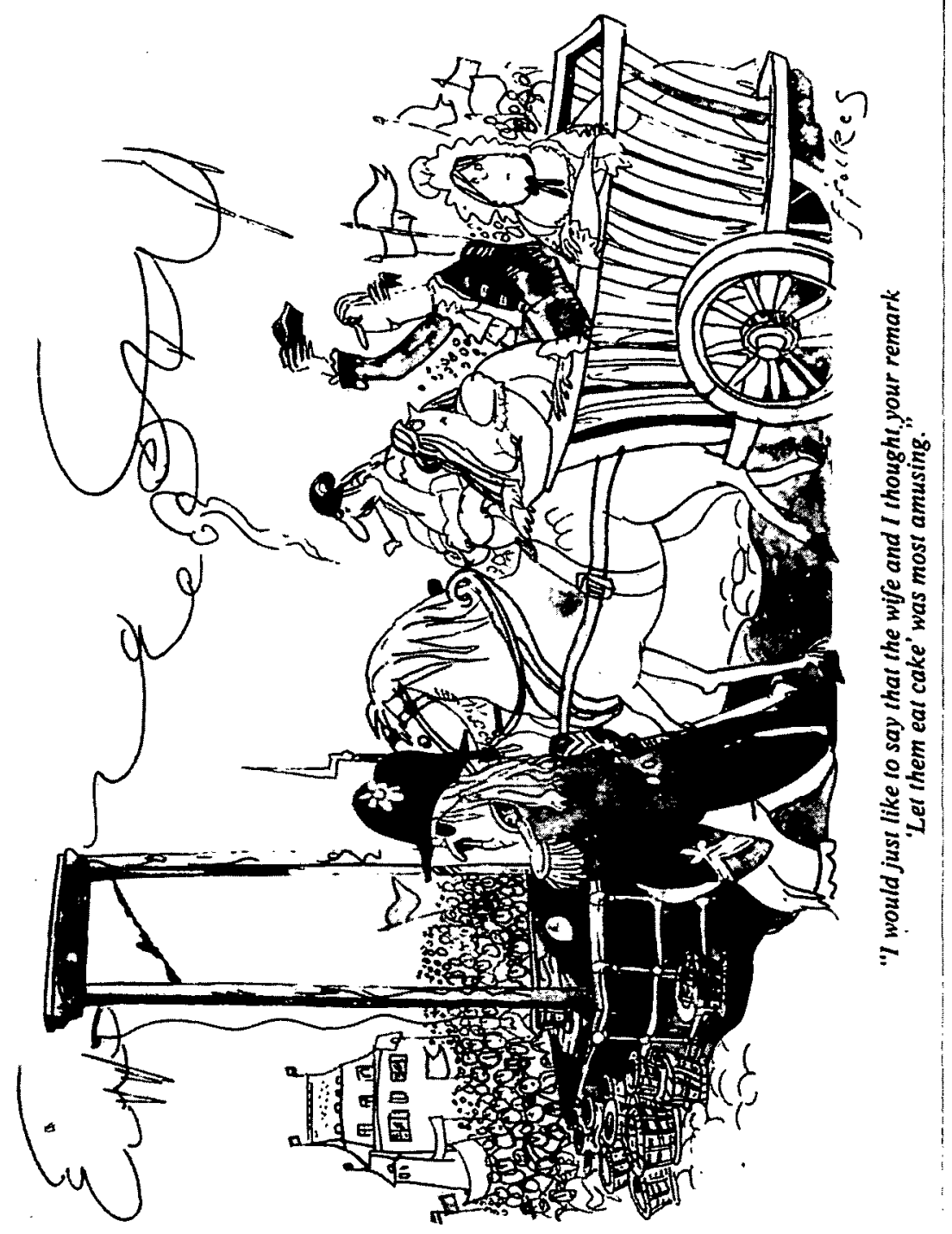

Fig. 3 : «I would just like to say that the wife and I thought your remark "Let them eat cake" was most amusing », Punch (29 octobre 1980) 742 (collection Wolfgang Mieder) 

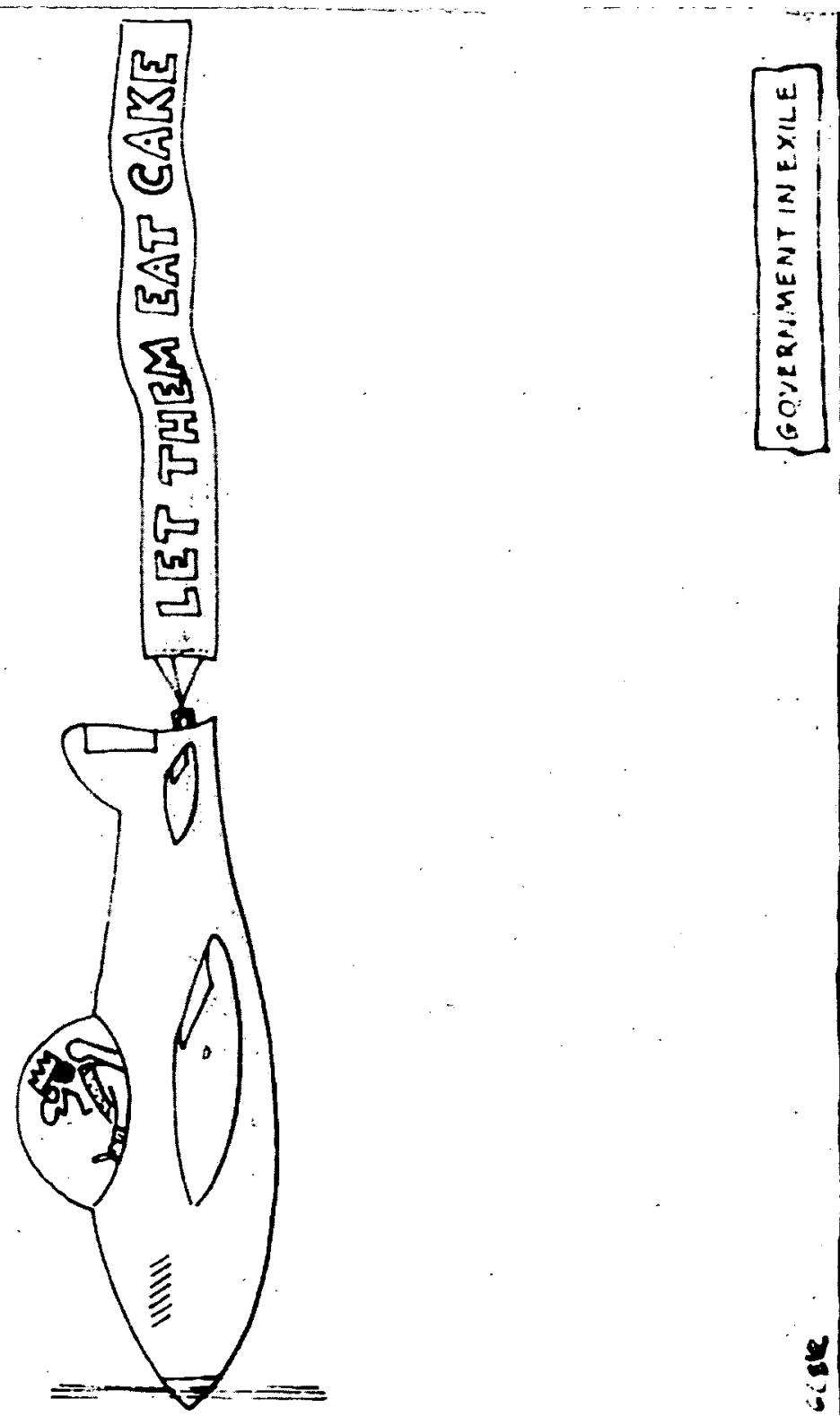

Fig. 4 : «Let them eat cake. Government in exile»,

The New Yorker (21 décembre 1987) 37 (collection Wolfgang Mieder) 
Les jeux de mots des journalistes penchent vers la polémique politique : «Qu'ils mangent des hors-d'œuvres» est le titre d'un reportage de Newsweek qui suggère que l'élite nord-coréenne festoie tandis que le pays connaît la famine. L'article conclut : «Le grand leader vénéré leur donnera peut-être du chocolat» (64). Des travailleurs protestataires de chez Renault symbolisant leurs besoins en défilant avec des demi-baguettes dans la bouche, la légende de la photo publiée dans Newsweek est (évidemment) : «Qu'ils mangent du pain » (65).

Des répliques proches du dire de la brioche sont attestées dans les régions arabophones dès les $\mathrm{XI}^{\mathrm{e}}-\mathrm{XIII}{ }^{\mathrm{e}}$ siècles. Bien qu'elles soient présentes dans des compilations de textes comiques, elles ont peut-être commencé sérieusement - tout comme les anecdotes chinoises citées plus haut (66) car elles évoquent des disettes et des riches vivant sur un très grand pied : Comme on lui annonce que le prix de la farine a beaucoup augmenté, un sot déclare : «Cela m'est égal. Je ne cuis pas le pain. Je l'achète » (67).

Dans l'ensemble, il semble qu'il y ait autant d'applications moralisatrices, didactiques ou accusatoires que d'exemples purement d'intention plaisante. En même temps, il y a un remarquable jeu entre des formes narratives distinctes. Un exemple frappant est la réplique cruelle «Qu'ils mangent donc du foin », liée à la rumeur politique dans la France révolutionnaire et à la tradition moralisatrice germanique des exempta, qui se retrouve dans un conte merveilleux galicien du $\mathrm{XX}^{\mathrm{e}}$ siècle sous la forme d'une dure réplique des deux méchants frères du héros à la merveilleuse figure secourable :

«Il croisa une femme tenant un enfant en pleurs dans les bras. Elle l'arrêta et lui dit "Jeune homme, me donneras-tu une croûte de pain pour cet enfant que la faim fait pleurer ?" "Qu'il mange de la paille", répondit le jeune homme. [La scène se répète quasi à l'identique lorsque le second frère rencontre la femme] » (68).

Eat Cake, London, ca 1961 (comedie, copyright 1938 sous le titre « Once Is Enough ») ; le téléfilm de la BBC «Let Them Eat Cake» (6 épisodes, diffusé du 9 septembre au 14 octobre 1999); George et Ira GERSHWIN, Let 'Em Eat Cake (première représentation à New York en 1933), chansons « Let 'Em Eat Cake, Let 'Em Eat Caviar »; Motorpsycho (groupe de rock norvégien) «Let Them Eat Cake », CD (Columbia 4974642, 2000) ; "Clinton Administration Says "Let Them Eat Cancer" » (communiqué de presse du Government Accountability Project, 3 décembre 1999, affirmant que volailles et bétail contenant des parasites, des tumeurs ou des abcès etc. entrent dans la chaîne alimentaire) (www.whistleblower.org/ www/eatcancer.htm) ; Garry HAMILTON, « Let Them Eat Dirt », New Scientist, 18 juillet 1998 (sur les désavantages possibles de l'obsession moderne de la propreté et de l'hygiène) (www.newscientist.com/us/ 980718/features.html) ; Arianna HUFFINGTON, «Let Them Eat Stock Options. The Democrats and RepubUcans have shamelessly abandoned the poor» (www.salon.com/news/feature/ 1999/09/16/poverty/).

(64) Newsweek (6 octobre 1997), 23 ; voir aussi « Hungerproblem gelöst : einfach mehr spachteln »

Titanic. Das endgiiltige Satiremagazin, 1980, 12, couverture.

(65) Newsweek (1 $1^{\mathrm{er}}$ décembre 1997) 17.

(66) Voir partie 2.

(67) Ulrich MARZOLPH Arabia ridens. Die humoristische Kurzprosa der frühen adab-Literatur im internationalen Traditions seflecht 2, Frankfurt am Main, 1992, $\mathrm{n}^{\circ} 496$.

(68) Marisa REY-HENNINGSEN, The Tales of the Ploughwoman [FF Comunications 259], Helsinki, 1996, n 24. 


\section{Trois modes accusatoires}

Le dire choquant est expliqué selon trois modes accusatoires : la bêtise ou l'ignorance, attribuées surtout à des femmes ; la cruauté outrageante, qui met en scène des hommes.

On rencontre dans la littérature populaire et le folklore, de nombreux textes où les femmes sont présentées de façon stéréotypée comme stupides ou simples d'esprit. L'épouse de Louis XIV, Marie-Thérèse d'Autriche et leur arrière-arrière-petite-fille, Madame Victoire, avaient une réputation de stupidité et la remarque «Mais, mon Dieu, s'ils pouvaient se résigner à manger de la croûte de pâté ! », lorsqu'elle leur est attribuée par un membre de la famille royale (le roi Louis XVIII) ou par des membres de leur entourage (la comtesse de Boigne) (69), semble une plaisanterie faite à leurs dépens. Il en va différemment lorsque des remarques similaires sont citées par des classes inférieures, comme dans les exempta publiés par des érudits allemands du XVI siècle, dans des contes lettons et finnois, un almanach hollandais (70) ou l'évocation irlandaise d'une supposée tradition italienne :

« Il y a à Superega, Superga, la tombe d'une princesse du Piémont qui se rendit célèbre par une seule phrase. Une famine sévissait dans ce petit royaume. La princesse était stupéfaite "En meurent-ils?" demanda-t-elle. "En grand nombre" lui répondit-on. "Quelle pusillanimité" déclara Son Altesse ; "Pourquoi ne mangent-ils pas du bœuf et de l'agneau ? C'est, j'en suis sûre, ce que je ferais au lieu d'avoir faim". » (71)

Se combinant avec l'intention de ridiculiser la classe supérieure, ridiculiser, les femmes devient donc une expression de critique sociale, un moyen de détourner l'agression, et peut aussi être utilisé comme une arme par des agitateurs politiques.

Lorsque celle qui a prononcé la célèbre réplique est présentée comme simplement ignorante, elle est pardonnée, tout au moins jusqu'à un certain point : elle n'est pas véritablement stupide, simplement un peu sotte et pas véritablement coupable, ce sont les circonstances qui sont à blâmer. Erich Kaestner affirme que Marie-Antoinette ne connaissait pas les conditions de vie du peuple; de même Henry de Montherlant remarque dans Les célibataires:

«On a voulu que le dire de Marie-Antoinette : "S'ils n'ont pas de pain, qu'ils mangent de la brioche", fût un mot odieux. C'était peut-être, simplement, que Marie-Antoinette croyait que la brioche coûtait le même prix que le pain ». (72)

(69) Mémoires de la comtesse de Boigne, éd. Jean-Claude BERCHET, Paris, 1982, p. 55.

(70) Provinciale Groninger Hazelhoff's Almarmk, voor het jaar 1874, Groningen, 1873, s. p.

(71) Ireland's Own 5, 105, 30 novembre 1904, 1.

(72) Henry de MONTHERLANT, Les célibataires, Paris, 1934, 1945, p. 53. 
Une variante américaine attribue la réplique à l'ignorance enfantine de la fille de Marie-Antoinette (73), et Madame Victoire, qui passait pour simplette, proféra sa remarque, suivant Madame de Boigne, avec des larmes aux yeux; Madame de Boigne déclare également :

«Pour moi, j'y crois, d'abord parce que ma mère m'a dit que madame Adélaïde en plaisantait souvent sa sœur qui avait horreur de la croûte de pâté [...], et puis parce que j'ai encore vu et su tant de traits de cette ingénuité vraie et candide sur la vie réelle que cela m'étonne beaucoup moins que la génération nouvelle. » (74)

C'est comme un signe de l'ignorance de la duchesse du Maine que le compilateur de la Correspondance secrète, politique et littéraire citant Madame de Staal-Delaunay lui attribue la réplique en 1788 :

«Elle ne se soucie point d'être entendue; il lui suffit d'être écoutée. Aussi n'a-t-elle aucune connaissance de l'esprit, des talens, des défauts \& des ridicules de ceux qui l'environnent. On a dit d'elle qu'elle n'était point sortie de chez elle \& qu'elle n'avait pas même mis la tête à la fenêtre [en note, on prétend en effet qu'un jour sur ce qu'on lui disait que les paysans n'avaient point de pain, elle s'écria de bonne foi : "Eh bien, qu'ils mangent de la brioche"]. » (75)

Bien qu'ouvertement la méchanceté soit absente des exemples de bêtise ou d'ignorance, un trait de critique sociale visant l'indifférence et l'ignorance des riches est toujours présent, même discrètement.

Conformément au stéréotype misogyne des femmes sans cervelle, ces accusations de bêtise ou d' ignorance ne mettent en scène en Europe que des protagonistes féminines (76), mais lorsque la cruauté entre en jeu, les hommes apparaissent aussi, voire surtout. Dans la France du xviiie siècle, des membres de l'élite maléfique censée organiser le pacte de famine avaient, affirmait-on, recommandé de façon moqueuse aux affamés du foin, de l'herbe ou des trognons de chou, c'est-à-dire des aliments destinés aux animaux. En 1725, année de quasi-famine, une attestation ancienne cite l'intendant de police de Paris, Ravot d'Ombreval, (parent de Madame de Prie, maîtresse du Premier ministre, le duc de Bourbon, accusés tous deux d'organiser la rareté des céréales) :

« Mis en présence, à la Halle, d'une mère qui gémit de ne pouvoir nourrir son enfant "le pain étant hors de prix", le lieutenant [d'Ombreval] conseille de lui donner "des trognons de choux". » (77)

(73) TAYLOR, op. cit., 4.

(74) DE BOIGNE, op. cit., p. 371

(75) «Portrait de Madame la Duchesse du Maine par Madame de Stal. », Correspondance secrète, politique et littéraire ou Mémoires pour servir à l'histoire des cours, des sociétés et de la littérature en France depuis la mort de Louis XV, Londres, John Adamson, 1788, tome XIV, pp. 150-154. citation p. 153. ignorants.

(76) Notons toutefois qu'en Inde et en Chine (voir page 37) apparaissent des hommes stupides ou

(77) KAPLAN, op. cit., pp. 19 et suiv. 
Un complice de la famine est ici montré se gaussant des pleurs de la faim.

Pendant la Réforme, les auteurs allemands employaient une réplique encore pire «Qu'ils mangent de la merde» afin de démontrer qu'une conduite scandaleuse et sans cœur serait punie par Dieu. Ce châtiment céleste est d'autant plus sans équivoque et impressionnant qu'il suit la loi du talion : suivant sa réplique, la méchante noble fut condamnée à demeurer affamée et finalement fut réduite à l'état d'un ver - elle commença à manger de la terre, puis du crottin et finalement des excréments humains (78). Cette tradition des exempta trouve un écho dans une légende poméranienne de la première moitié $\mathrm{du} \mathrm{XIX}^{\mathrm{e}}$ siècle qui montre une princesse qui jette chaque jour de grandes quantités de poisson. Pendant une famine, les gens du peuple affamés viennent l'implorer de leur donner du pain, mais elle les fait chasser avec le fouet à chien et fait jeter de grandes quantités de pain et de farine. Châtiment céleste, un éclair la tue et réduit la ville en cendres. Certes la réplique cruelle n'est pas prononcée, mais l'esprit de la légende est voisin de notre dire (79).

Dans le climat pré-révolutionnaire marqué d'émeutes de la faim, ces répliques sont fréquemment imputées à des puissants, dont un gouverneur militaire de Dijon, La Tour du Pin :

«Il est peu probable que le commandant militaire de Dijon, La Tour du Pin, ait dit aux paysans affamés d'aller brouter l'herbe qui commençait à pousser, car c'est là un de ces mots en quelque sorte traditionnels que l'on retrouve reproduit sous une forme ou sous une autre dans tous les soulèvements analogues. » (80)

Le cas le plus connu est l'attribution au financier et ministre JosephFrançois Foulon, pour lequel l'assertion eut des conséquences bien réelles et terribles. Son lynchage, quelques jours après la prise de la Bastille en 1789, imita en effet la réplique qui lui était attribuée. Financier, Foulon qui fût envisagé comme ministre des Finances sous Louis XV, fut successivement intendant militaire puis naval, et conseiller. Le 12 juillet 1789, peu avant la prise de la Bastille, il devint contrôleur général des Finances. Son gendre, Berthier était l'intendant de Paris. Le portrait fort défavorable des deux hommes tracé par Michelet - qui les présente comme des réactionnaires assoiffés de sang et des trafiquants douteux sur les blés participant au pacte de famine - semble justifier leurs morts terribles par lynchage

(78) Recueils de Jobum FINCELIUM, Leipzig, 1559 , Andream HONDORFF, Leipzig, 1568 ; M. Casparum TITIUM Pfarrherrn Zu Heckstet, Wittenberg, 1657.

(79) J. D. H. TEMME, Die Volkssagen von Pommern und Rügen, Berlin, 1840, n 164.

(80) La Grande Encyclopédie 17, Paris, 1904, p. 19. Philippe-Antoine-Gabriel-Victor de la Tour du Pin-Gouvernet, marquis de la Charce (ca 1723-94 [guillotiné]) était commandant militaire de Bourgogne lors de la Révolution. Voir Henri BEAUNE, Jules D'ARBAUMONT, La noblesse aux États de Bourgogne de 1350 à 1789, Dijon, 1864, p. 311. Voir aussi LAROUSSE, op. cit., $t$. 10, 1873, pp. 242 et suiv. 
dans les mains d'une foule furieuse le 22 (23 ?) juillet 1789 (81). Michelet donne deux versions de la cruelle réplique attribuée à Foulon, une réplique pire que celle de d'Ombreval car elle traite la foule affamée comme un troupeau d'animaux, de bêtes brutes: "S'ils ont faim, qu'ils broutent l'herbe [...]. Patience! Que je sois ministre, je leur ferai manger du foin ; mes chevaux en mangent [...] » (82). Suivant une autre version, Foulon aurait dit : « Je les réduirai à manger du pain à 5 sous la livre ou à se nourrir du foin » (83). La seconde version rapportée par Michelet est : «On lui imputait encore ce mot terrible: "Il faut faucher la France [...]" » (84) [On trouve également: «On devrait faucher Paris comme on fauche un pré » (85); "Je ferai faucher Paris comme un pré »] (86). Dans sa forme la plus répandue la réplique est plus neutre, bien qu'elle animalise la foule : «Eh bien! si cette canaille n'a pas de pain, elle mangera du foin » (87); «Si le pain manque, que le peuple mange du foin. » (88)

Foulon et Berthier se cachèrent après la prise de la Bastille. On dit même que Foulon tenta de passer pour mort tandis que Berthier s'enfuit vers le Nord, mais fut arrêté à Soissons. Suivant Michelet, c'est à la maison d'un ami, le lieutenant de police de Paris Sartine, que Foulon fut arrêté et conduit à Paris. « Ils trouvèrent le mort, qui se promenait bien-portant dans le parc de M. de Sartine: "Tu voulais nous donner du foin, c'est toi qui en mangeras !" On lui met une botte de foin sur le dos, un bouquet d'orties, un collier de chardons » (89). À l'Hôtel de Ville, il fut traîné dans la rue par une foule furieuse et pendu à un réverbère. «Foulon est enlevé, porté à la lanterne d'en face ; on lui fait demander pardon à la nation. Puis hissé... Par deux fois, la corde casse. On persiste, on va en chercher une neuve. Pendu enfin, décapité, la tête portée dans Paris [...] livide et du foin dans la bouche » (90). La cruelle réplique qui lui était attribuée illustra donc son meurtre exemplaire. Dans d'autres villes, plusieurs victimes, des boulangers surtout, furent tués ces jours-là par des foules lors d'émeutes de la faim. La tête trophée de Foulon fut présentée à Berthier afin qu'il l'embrasse ; lui-

(81) Les attestations écrites renvoient au 22 juillet, mais une gravure du musée Carnavalet est légendée 23 juillet.

(82) MICHELET, op. cit., pp. 183-184. Le massacre de Foulon et Berthier et les événements le précédant sont décrits, pp. 177-189.

(83) Dictionnaire de biographie française 14, Paris ,1979, pp. 671-673.

(84) MICHELET, op. cit., p. 184.

(85) Dictionnaire de biographie française 14 , op. cit., p. 672.

(86) La Grande Encyclopédie 17, op. cit., p. 893.

(87) LAROUSSE, op. cit., t. 8, p. 660.

(88) La Grande Encyclopédie 17, op. cit., p. 893.

(89) MICHELET, op. cit., p. 186.

(90) MICHELET, op. cit., pp. 186-187. 
même fut peu après tué par la foule ; son cœur arraché jeté aux autorités de la ville (91).

La cruelle réplique est fréquente dans les émeutes de la faim, comme le remarque Georges Lefebvre :

« À Lons-le-Saulnier, deux membres du Parlement furent accusés "d'avoir voulu faire manger de l'herbe au peuple"; à Sainte-Maure, en Touraine, Turquand, procureur du roi de la municipalité et son fils furent inculpés de propos insultants : "Que les gueux de paysans seraient obligés de manger de l'herbe et des racines pour vivre, de faire faire de la bouillie à leurs enfants avec de la raclure de pierre blanche et que les têtes fontangées ne mangeraient pas leur saoûlt de pain d'orge." À Orléans, en l'an II, un ancien échevin fut arrêté pour avoir, racontait-on, observé en 1789 que, "si les petites filles mouraient, il y aurait assez de pain", propos que d'autres grossirent de la manière suivante : "qu'il fallait jeter les enfants à la rivière parce que le pain était trop cher". » (92)

L'histoire montre d'étonnantes permanences, et le modèle d'extrême cruauté fonctionnait toujours au XIX ${ }^{\mathrm{e}}$ siècle dans la situation d'oppression vécue par les Indiens Sioux aux États-Unis. Leur révolte de 1862 semble avoir été déclenchée, entre autres, par l'insultante réflexion d'un commerçant, Andrew J. Myrick, qui aurait déclaré «Pour moi, s'ils ont faim, qu'ils mangent donc de l'herbe ou leur merde » (93). Dans sa réponse au colonel Sibley, le chef indien Little Crow cita la réplique «comme un des motifs de son entrée en guerre, nommant le commerçant, et citant sa réflexion en y ajoutant une variante (94). Myrick fut un des premiers à être abattu par arme à feu le matin de la révolte, et lorsque son corps fut retrouvé par les hommes de Sibley sa bouche était remplie de l'herbe qu'il avait recommandée aux Indiens comme nourriture. » (95)

\section{Utilisation didactique et politique du dire de Marie-Antoinette de nos jours}

Tout comme les répliques cruelles, l'exemple d'ignorance de MarieAntoinette a souvent été utilisé de façon moralisatrice. Erich Kaestner, qui s'adresse aux enfants afin de leur enseigner des valeurs morales telles que la justice, l'égalité et la responsabilité sociale, met l'accent sur les espoirs pour

(91) Autres descriptions dans LAROUSSE, op. cit., t. 8, p. 660; La Grande Encyclopédie 17, op. cit., p. 893 ; Dictionnaire de biographie française 14, op. cit., pp. 671-673; HOEFER, Nouvelle biographie générale 18, Paris, 1857, pp. 297 et suiv. ; M. A. THIERS, Histoire de la Révolution française 1, Paris, 1834, pp. 125-127 (ne mentionne pas la réplique attribuée à Foulon).

(92) LEFEBVRE, op. cit., p. 34

(93) Duane SCHULTZ, Over the Earth I come. The Great Sioux Uprising of 1862, New York, 1992, p. 28 et William Watts FOLWEU, History of Minnesota, Saint Paul, 1924, vol. 2, p. 233. Cette deuxième source cite «qu'ils mangent donc de l'herbe ».

(94) Sans doute la référence aux excréments.

(95) FOLWEL, op. cit. 
l'avenir et non sur les désirs de vengeance. Après l'anecdote de MarieAntoinette, il poursuit :

«Ne croyez-vous pas que la pauvreté pourrait être plus facilement supprimée si les riches savaient, dès leur enfance, combien il est dur d'être pauvre? Ne croyez-vous pas que les enfants riches se diraient alors : quand nous serons grands, quand nous posséderons les banques, les terres et les usines de nos pères, nous travaillerons à ce que les ouvriers soient plus heureux. enfance.

Car les ouvriers, ce seraient leurs camarades de jeu du temps de leur

Croyez-vous que cela soit possible?

Voulez-vous aider à ce que cela se réalise ? » (96)

CSK avait emprunté Pünktchen und Anton à la bibliothèque et sous ces lignes, un enfant avait écrit Oui! avec un gros point d'exclamation. Lorsqu'elle lut le livre à sa fille, Charlotte répondit à ce passage par un oui ! spontané, et bien d'autres enfants feraient de même.

Pendant ses recherches en 1997, VCV trouva deux exemples de transmission orale de la célèbre réplique de Marie-Antoinette à des enfants :

Dans un contexte laïque et parisien, R.B. (maintenant professeur d'histoire moderne à l'Université d'Arras) avait entendu l'anecdote en classe en 1954, lorsqu'il avait 9 ans. C'était son instituteur malgache qui avait narré cette histoire morale en classe comme expliquant la Révolution par la dureté de l'aristocratie française, dont l'exemple parfait était la réplique de Marie-Antoinette. R.B. s'en souvenait car sa mère lui avait dit trouver l'anecdote invraisemblable (97).

Dans un contexte catholique et provincial (Angers, dans l'Ouest contrerévolutionnaire), F.A. (maintenant journaliste culturel) entendit l'anecdote en 1968, lorsqu'il était âgé de 10 ans. Elle était attribuée à Marie-Antoinette et lui fut racontée dans un cadre familial par une tante, professeur de couture âgée d'environ 70 ans. La réplique était présentée comme un exemple de la dureté de cœur et des abus des grands, causes des révolutions (98).

Énoncées dans des contextes sociaux et idéologiques opposés, ces deux communications orales semblent avoir partagé la même intention didactique d'explication de la Révolution. Toutefois la similarité n'est que superficielle, car l'instituteur voulait glorifier une Révolution juste tandis que la tante voulait déplorer ses désordres et ses maux.

(96) KAESTNER, op. cit., p. 66. Traduction révisée par CSK.

(97) Communication personnelle à VCV, 17 juin 1997.

(98)Id., 16 juin 1997. 
Dès que des sujets politiques, éthiques ou sociaux apparaissent, la formule «Qu'ils mangent de la brioche » surgit spontanément (99).

Ainsi dans la controverse à propos des organismes génétiquement modifiés (OGM), une discussion opposant adversaires et partisans de cette technologie se tint en 1997 sur l'Internet sous la référence «Qu'ils mangent de la brioche » (100).

En 1999, un article du Asian Wall Street Journal accusait les éco-activistes d'« exporter leur paranoïa vers les pays en développement qui ont le plus besoin de récoltes génétiquement modifiées ». Son auteur opposait les intérêts du «petit fermier en Inde, dont les récoltes sont détruites par les maladies ou les insectes» et ceux d'une «aristocratie sans problème de subsistance », (une allusion à Lord Peter Melchett, directeur exécutif de Greenpeace U.K.), il stigmatisait pour conclure le point de vue des écologistes « une attitude complètement "Qu'ils mangent de la brioche" » (101).

Plus étroitement associée à Marie-Antoinette, la célèbre réplique s'utilise également dans les discussions politiques menées dans la presse française. Fin 1999, Le Monde indiqua que des Britanniques membres du Parlement européen l'avaient utilisée afin de protester contre la politique européenne dans l'affaire de la vache folle (102). Elle semble spécialement liée, toutefois, à la question de l'immigration. Récemment, dans le contexte de la politique scolaire, la suggestion que l'apprentissage du latin et du grec par les enfants maghrébins pouvait aider à leur intégration a été sarcastiquement comparée au dire de la brioche :

« Il y a comme une tartufferie à vouloir persuader les enfants d'immigrés maghrébins qu'il leur suffit "d'apprendre le latin et le grec" pour saisir leur "meilleure chance d'intégration". Marie-Antoinette n'aurait rien trouvé à redire, qui suggérait des brioches aux pauvres qui manquaient de pain. » (103)

Lorsqu'un hagiographe de Marie-Antoinette, à l'occasion du bicentenaire de sa mort, l'appelle immigrée, la brioche est appelée à la rescousse pour exprimer l'indignation d'un chroniqueur :

«Jean Chalon, donc, écrivait l'autre semaine dans son journal [Le Figaro] qu'on en voulait à Mme Capet parce qu'elle était "immigrée". On a bien lu : "immigrée", comme un quelconque OS marocain de chez Renault.

(99) Voir les exemples de la partie 3.

(100) Lettres des 3 et 4 août 1997, www.gene.ch/gentech/1997/Jul-Aug/msg00381.html et www.gene.ch/gentech/1997/Jul-Aug/msg00403.html, www.biotech-info.net/eat-cake.htm.

(101) Michael T. WILSON, « Eco-activists to the Poor : "Let Them Eat Cake" », Asian Wall Street Journal, 9 mars 1999.

(102) «Mardi 14 décembre, cinquante euro-députés britanniques déployaient aux portes du Parlement de Strasbourg une banderole en anglais: "Qu'ils mangent de la brioche : Marie-Antoinette1789. Qu'ils mangent du boeuf britannique: députés britanniques-1999." », Le Monde, rubrique «Kiosque », 17 décembre 1999.

(103) Saad KHIARI, «Absences arabes », Le Monde, 9 mars 2000, p. 16. 
Voilà les brioches de Versailles et les beurs de La Courneuve dans le même panier. » (104)

En 1997, Alain Finkielkraut utilisa la réplique de Marie-Antoinette en critiquant les intellectuels qui luttaient contre une loi de contrôle des immigrés. Il jugeait que les intellectuels, trop élitistes, ignoraient des plaintes authentiques et justifiées des couches populaires envers les immigrés dont la présence aggravait les conditions de vie des pauvres :

«Comment ce discours peut-il être reçu par les victimes économiques? Comme celui de Marie-Antoinette lançant au peuple affamé : "Vous manquez de pain, mangez donc de la brioche !" Mon inquiétude, depuis les années Mitterrand, tient au fait que c'est Marie-Antoinette qui mène le combat antiraciste. [...] Les enfants gâtés de l'époque, les privilégiés, qui évoluent dans la mondialisation comme des poissons dans un bocal, ne sont pas les mieux à même de faire la leçon à ceux qui sont hors du bocal. » (105)

La réplique à un tiers prêtée à la reine devient ici dialogue direct et Marie-Antoinette est présentée comme agressive et cynique envers la foule affamée, lançant un défi à ceux qu'elle dédaigne plutôt que faisant preuve de bêtise ou d'ignorance. Les lecteurs indignés rejetèrent vigoureusement l'attribution à Marie-Antoinette : "J'ai lu avec consternation l'article du Figaro Magazine du 22 février, dans lequel Alain Finkielkraut, que j'ai toujours tenu pour un homme érudit, accrédite la phrase-bidon attribuée successivement à Mme de Pompadour, Mme du Barry, et MarieAntoinette » (106). On le voit, même des érudits participent, consciemment ou inconsciemment, à la communication de niveau moyen lorsqu'ils sortent de leur sphère spécialisée, alors que dans ce cas les spécialistes, les historiens de la Révolution française et de la période la précédant ne disent rien et ignorent cette réplique et sa célébrité.

Finalement, trois exemples nous montrent des usages politiques de la célèbre réplique. Ici, l'accusé n'est pas Marie-Antoinette, nous renvoyant aux aristocrates et aux super-riches, mais l'État lui-même, ignorant le sort difficile de ses sujets dans son exercice froid du pouvoir.

Le premier exemple est littéraire et remonte à 1933. Dans Miss Lonelyhearts, du romancier américain Nathanael West, le protagoniste, qui assure la rubrique courrier personnel d'un quotidien new-yorkais, reçoit le conseil de suggérer à ses correspondants la révolte comme unique solution pour remédier à l'injustice de l'ordre des choses :

(104) François REYNAERT, « Requiem pour un bicentenaire : Marie-Antoinette et les MarieChantal », Le Nouvel Observateur, 21-27 octobre 1993, p. 108.

(105) Sylvie PIERRE-BROSSOLETTE, «Le philosophe Alain Finkielkraut : "Marie-Antoinette défend les immigrés" » Le Figaro Magazine, 22 février 1997, pp. 15 et suiv., ici p. 16.

(106) Marie-Claude MONCHAUX, lettre au Figaro Magazine, 8 mars, 1997, p. 7. Un autre lecteur, Jean Avré (8 mars 1997), ajouta que la phrase avait été écrite par «l'immigré » Rousseau. 
«Miss Lonelyhearts, mon ami, je te conseille de donner des pierres à tes lecteurs. Quand ils te demandent du pain, ne leur donne pas des biscuits comme l'Église, ne leur dit pas comme l'État, de manger de la brioche. Apprends-leur à prier chaque matin: "Donnez-nous notre pierre quotidienne". »(107)

Le second exemple est tiré d'un document canadien de protestation politique, un tract militant, et il est contemporain. Ce texte a été enregistré pendant l'interview de Christian, chômeur de 31 ans, étudiant du Québec, en 1998 :

«On a l'impression que le ministre dit comme Marie-Antoinette : "Ils ont pas de pain, ben qu'ils mangent du gâteau [!], c'est le destin. On a même pas de farine." Les économistes devraient faire attention à l'histoire parce qu'ils font exactement la même lutte de privilèges que les monarchistes ont faite en disant: "Je suis le seigneur et maître de ces lieux. Tu es un serf, tu restes un serf." [...] Les gens d'affaires aujourd'hui, qui étaient les bourgeois révolutionnaires de l'époque, font la même chose que les monarchistes. » (108)

Le troisième exemple, également contemporain, vient d'une lettre de lecteur, publiée dans Le Monde à l'occasion du débat sur la loi Aubry contre l'exclusion, dont les mesures d'accompagnement semblaient insuffisantes, inefficaces et condescendantes, à l'auteur qui se présentait comme "chômeur", c'est-à-dire membre du groupe des exclus concerné par la loi. Il utilise deux fois l'allégorie de la figure négative de Marie-Antoinette, dont il rapproche la "hautaine" Martine Aubry:

«"Humble supplique à Martine Aubry" par Jean-Claude Marcus, chômeur. La ministre Martine Aubry aurait-elle une lointaine parenté avec Marie-Antoinette ? Faute de brioche - la Cour pourrait en manquer - elle fera distribuer au bas peuple quignons de pain par millions et même petites corvées par dizaines de milliers [...]. Parmi tant d'autres voici, présentées humblement, en forme de supplique, trois menues doléances à MartineAntoinette. »(109)

\section{Conclusion}

En son temps, l'image fort négative de Marie-Antoinette se retrouva dans les surnoms de Madame Déficit ou Madame Véto. L'extravagance et la prodigalité de la reine, sa vie privée, étaient la cible d'innombrables chansons, satires et pamphlets. Bien qu'innocente, elle fut impliquée dans la

(107) The Complete Works of Nathanael West, London 1983, pp. 70 et suiv.

(108) Carrefour de pastorale en monde ouvrier «Carrefour de savoirs sur les finances publiques : Le niveau de vie de neuf personnes en situation de très grande pauvreté dans les quartiers centraux de Québec », Québec 1999.

(109) Le Monde, 10 mars 1998. 
célèbre « affaire du Collier » qui aggrava encore sa réputation désastreuse. Mais avec le temps, les faits politiques et personnels complexes derrière tous ces ragots, cette satire, ce persiflage et ces calomnies devinrent trop compliqués pour être mémorisés par les générations suivantes. Par contre la réplique «Qu'ils mangent de la brioche », simple et directe, aisément retenue, devint une sorte de sommaire des grands maux de l'âge pré-révolutionnaire : la famine et l'exploitation des masses; la frivolité, l'irresponsabilité et la morgue de la noblesse. Dans un processus similaire, les autres individus auxquels cette réplique ou des répliques analogues étaient prêtées autrefois ont, petit à petit, disparu de la mémoire collective où seules quelques figures symboliques d'importance pouvaient garder leurs places.

Dans l'esprit populaire, le cours de l'histoire ne subsiste que de façon rudimentaire, la confusion et la complexité des faits et événements, des idées et courants sont épurés pour former un tout logique, significatif et cohérent, les multiples acteurs sont réduits à quelques personnages clés. Et ainsi, jusqu'à ce jour, le souvenir de Marie-Antoinette est couramment caractérisé par cette brève réplique : «Qu'ils mangent de la brioche ! »

Véronique CAMPION-VINCENT

Maison des Sciences de l'Homme

54 boulevard Raspail, 75008 Paris

campion@msh-paris.fr

Christine SHOJAEI KAWAN

Enzyklopàdie des Màrchens

Friedlander weg 2, D-3700085 Gôttingen

ckawan@gwdg.de 